\title{
Finite energy quantization on a topology changing spacetime
}

\author{
S. Krasnikov \\ Central Astronomical Observatory at Pulkovo, St.Petersburg, 196140, Russia
}

(Dated:)

\begin{abstract}
The "trousers" spacetime is a pair of flat 2D cylinders ("legs") merging into a single one ("trunk"). In spite of its simplicity this spacetime has a few features (including, in particular, a naked singularity in the "crotch") each of which is presumably unphysical, but for none of which a mechanism is known able to prevent its occurrence. Therefore it is interesting and important to study the behavior of the quantum fields in such a space. Anderson and DeWitt were the first to consider the free scalar field in the trousers spacetime. They argued that the crotch singularity produces an infinitely bright flash, which was interpreted as evidence that the topology of space is dynamically preserved. Similar divergencies were later discovered by Manogue, Copeland and Dray who used a more exotic quantization scheme. Later yet the same result obtained within a somewhat different approach led Sorkin to the conclusion that the topological transition in question is suppressed in quantum gravity.
\end{abstract}

In this paper I show that the Anderson-DeWitt divergence is an artifact of their choice of the Fock space. By choosing a different one-particle Hilbert space one gets a quantum state in which the components of the stress-energy tensor (SET) are bounded in the frame of a free-falling observer.

\section{INTRODUCTION AND CONCLUSIONS}

The two-dimensional spacetime $\mathcal{M}$ called "trousers" is obtained from the strip

$$
\mathrm{d} s^{2}=\mathrm{d} t^{2}-\mathrm{d} x^{2} \quad t \in \mathbb{R}, \quad x \in[-P, P]
$$

by, first, deleting the points $t=0, x= \pm P$ and the ray $t \leq 0, x=\mu P$, then attaching a copy of the deleted ray to either bank of the cut and, finally, smoothly gluing each of the six rays to its counterpart so that the resulting space consists of three cylinders, see figure 1 . 


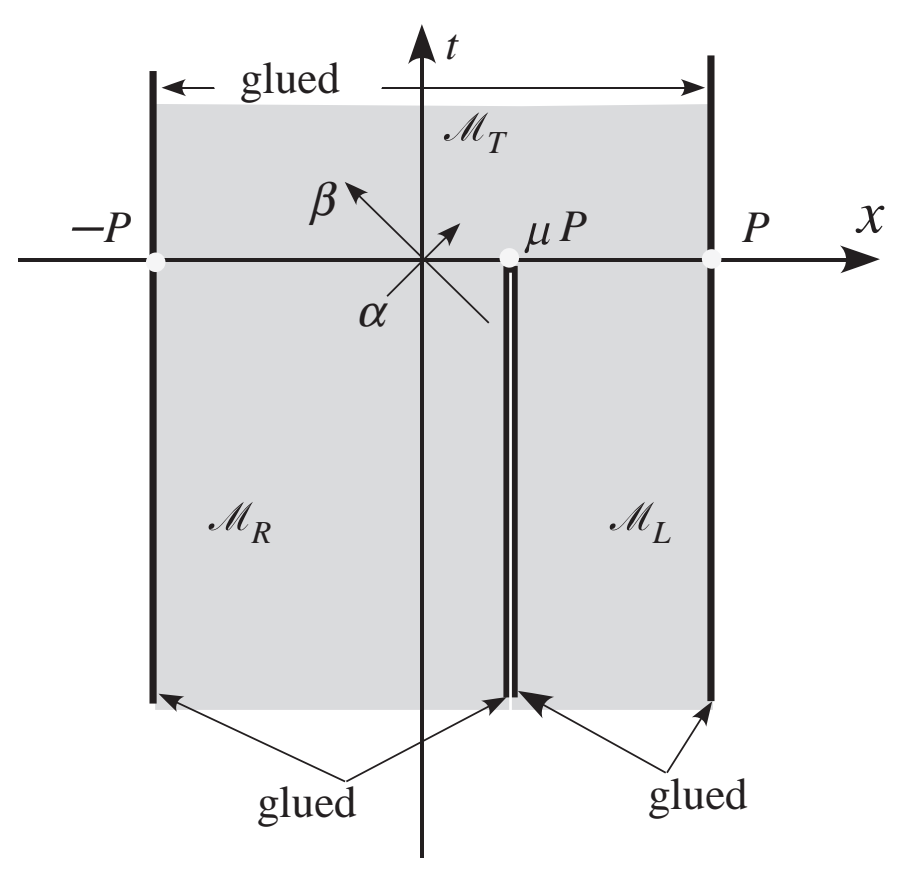

FIG. 1: Constructing the trousers spacetime from a flat strip. The left leg $\mathcal{M}_{L} \equiv(t \leq 0, x \in$ $[-P, \mu P))$, the right leg $\mathcal{M}_{R} \equiv(t \leq 0, x \in[\mu P, P))$, and the trunk $\mathcal{M}_{T} \equiv(t \geq 0, x \in(-P, P])$ are obtained by gluing together the rays bounding the corresponding strips. The white circles depict the removed points. They cannot be returned back after the relevant identifications are performed and thus a naked quasiregular singularity appears.

The trousers spacetime merits the most detailed consideration because in spite of its simplicity it possesses two features, interesting and important, but poorly understood:

1. the topology of its spacelike sections changes with time. It is $\mathbb{S}^{1} \sqcup \mathbb{S}^{1}$ at negative $t$ and $\mathbb{S}^{1}$ at positive. This type of topology change is particularly significant, because it may have to do with the appearance of a wormhole or (if the $t$-axis is directed to the past as in [1]) with the final stage of the wormholes (including the Schwarzschild black hole) evaporation [2];

2. the spacetime is singular, as one might expect, and the singularity-loosely speaking it is located at the "crotch of the trousers" - is naked and quasiregular. Presumably either of these properties makes it "unphysical", but no mechanism is found that would protect the Universe from the appearance of such singularities.

The evolution of a quantum field in trousers was first considered by Anderson and DeWitt (AD). In their well-known paper [1] they conjectured that the above-mentioned singularity 
emits an infinitely bright flash. Their reasoning was as follows [12] "[...] an 'in' mode function propagating to the right splits into components propagating to the right in each leg. Although continuous in the trunk region, such mode functions generally have discontinuities $[\ldots]$ in the legs. [...] Every 'out' mode function is continuous in each leg (vanishing in one of them) but has discontinuities in the trunk region. [...] When these functions are differentiated the discontinuities give rise to delta functions. Since the terms of the mode sum for $<$ in, vac $\left|\mathbf{T}_{\text {ren }}^{00}\right|$ in, vac $>$ are bilinear in differentiated mode functions, the square of the delta function automatically appears".

The divergence of $<$ in, vac $\left|\mathbf{T}_{00}^{\text {ren }}\right|$ in, vac $>$ does not automatically exclude the topology changes: it may happen that some of them are free from that divergence [3] or that the relevant quantity is the matrix element $<$ in, vac $\left|\mathbf{T}_{00}^{\mathrm{ren}}\right|$ out, vac $>$ as opposed to the expectation value of the stress-energy tensor [4]. It is also possible that the fields in such an unusual space must be quantized in some special way [5] (one such unusual quantization was proposed in [6], the resulting $\left\langle\mathbf{T}_{00}^{\text {ren }}\right\rangle$, though, diverges all the same). There is a good consensus, however, that the $\mathrm{AD}$ flashes are an indicator of some "flaw" in the trousers spacetime [1], [7], [9]. The goal of this paper is to show that this is not the case.

\section{Conclusions}

In regard to the divergence of the energy density the trousers turn out to be as "nice" as, say, the Schwarzschild space. The latter is not compromised by the fact that in some states (such as the Boulware vacuum) the energy density diverges at the horizon. What matters is the existence of states free of such divergences. Accordingly, we rehabilitate the trousers spacetime by explicitly constructing a state in which $\left\langle\mathbf{T}_{i k}^{\text {ren }}\right\rangle$ are bounded. Note that the existence of such a state does not contradict to the argument quoted above, owing to the word "generally" used in the latter. 


\section{THE QUANTIZATION}

\section{A. The plan}

The field $\phi$ considered in this paper obeys (classically) the wave equation

$$
\square \phi=0 .
$$

Though the spacetime under discussion is non-globally hyperbolic, the piecewise smooth (see below) complex-valued solutions of (1) are fixed uniquely - this is proven in section IIB - by the data at any surface $t=$ const $\leq 0$. In this sense the singularity is "harmless" [7, 8] and we can (and shall) proceed exactly as in the globally hyperbolic case. In doing so we are guided by the textbook [10], in particular, the units and sign conventions are those used there.

To canonically quantize the field one must first expand it as a series in vacuum modes that is find a set of functions $\left\{\phi_{k}\right\}$ on $\mathcal{M}$ that are an orthonormal basis in a Hilbert space $\mathcal{H}$. It is the choice of $\mathcal{H}$ that encodes the physics of the problem and determines the resulting theory.

The field operator $\hat{\phi}$ in QFT is taken to be an (operator-valued) distribution. But the Hilbert space in discussion is usually built on the basis of smooth solutions of the classical equation of motion (that is, $\mathcal{H}$ may contain non-smooth functions, but only those to which a sequence of smooth ones converges). Such a choice seems inadequate in studying "thunderbolts" with their discontinuities. Therefore Manogue, Copeland and Dray in [6] expanded the space by allowing the vacuum modes to have jumps. However, the derivatives of such modes will have $\delta$-like singularities exactly where the modes are discontinuous, which makes the Klein-Gordon scalar product ill-defined, see (2). Physically such singularities seem unwarranted too. Indeed, the crotch singularity can play the role of a source, so the solutions to the (now inhomogeneous) wave equation are expected to have irregularities on the null geodesics emanating from the "missing point". However, the energy density proportional to the square of the delta function is a too strong irregularity. So, in this paper we propose an intermediate approach and require classical solutions to be continuous, though not continuously differentiable. Specifically, let $\mathcal{C}$ be the space of bounded continuous complexvalued functions on $\mathcal{M}$ that are smooth solutions to equation (1) on the whole $X$ except perhaps at the points of past incomplete inextendible null geodesics (i. e., loosely speaking, 
null geodesics emanating from the singularity), where the derivatives of those functions may have jump discontinuities. Correspondingly, we are looking for a space $\mathcal{H}$ such that

1. $\mathcal{H}$ is a Hilbert space with respect to the Klein-Gordon scalar product

$$
\left.(f, g)_{\mathrm{KG}} \equiv i \int_{-P}^{P}\left(g^{*} \dot{f}-f \dot{g}^{*}\right)\right|_{t=0} \mathrm{~d} x
$$

(we indicate the particular value of $t$ because at this stage we cannot guarantee that the integral does not depend on the choice of that value, the spacetime being nonglobally hyperbolic). The positive-definiteness of this form is a non-trivial restriction on $\mathcal{H}$.

2. up to a constant any function $f$ in $\mathcal{C}$ is the sum of a function from $\mathcal{H}$ and a function from $\mathcal{H}^{*}$ :

$$
\text { for all } f \in \mathcal{C} \quad f=f^{+}+\left(f^{-}\right)^{*}+c, \quad f^{+}, f^{-} \in \mathcal{H}, \quad c=\text { const. }
$$

$f^{+}$and $\left(f^{-}\right)^{*}$ are often called, respectively, "positive and negative frequency" parts of $f$. Conversely, $\mathcal{H}$ must not include "superfluous functions", in other words, $\mathcal{H}$ must not have a proper subspace satisfying (3).

1. Remark. The word past in the definition of $\mathcal{C}$ signifies some time asymmetry in our approach which is not related to the asymmetry of the underlying spacetime.

\section{B. Reduction to initial conditions}

In this subsection we represent in a convenient form the space $\mathcal{C}$ : we use the periodicity of the functions constituting $\mathcal{C}$ to express them in terms of their restrictions to the surface $t=0$ (thus trading functions of two variables each for a pair of functions of one variable).

To begin with we note that any $\phi \in \mathcal{C}$ being a solution to the wave equation

$$
\partial_{\alpha} \partial_{\beta} \phi=0, \quad \alpha \equiv t+x, \quad \beta \equiv t-x
$$

is the sum of a right-moving and a left-moving (i. e. depending - within each of the cylinders - on the $\alpha$ - or, respectively, $\beta$-coordinate of its argument) function. Put more formally, it has the form

$$
\left.\phi\right|_{\mathcal{M}_{\curlywedge}}(p)=a_{\curlywedge}(\alpha(p))+b_{\curlywedge}(\beta(p))+c, \quad \curlywedge \equiv L, R, T .
$$


Here $c$ is an arbitrary constant and $a_{\curlywedge}(\alpha), b_{\curlywedge}(\beta)$ for each value of $\curlywedge$ are a pair of functions such that, first,

$$
a_{\curlywedge}(\alpha(p))=\left.a\right|_{\mathcal{M}_{\curlywedge}}(p), \quad b_{\curlywedge}(\beta(p))=\left.b\right|_{\mathcal{M}_{\curlywedge}}(p), \quad \text { where } a, b \in \mathcal{C}
$$

[note that the entire functions $a(p), b(p)$ do not have to be of the form $a(\alpha(p))$ and $b(\beta(p))$; moreover, they may have a discontinuity on the ray $t \leq 0, x=\mu P]$ and, second, they satisfy the following normalization conditions

$$
a_{L}(\mu P)=\frac{\int_{-P}^{\mu P} a_{L}(\alpha) \mathrm{d} \alpha}{(1+\mu) P}+\frac{\int_{\mu P}^{P} a_{R}(\alpha) \mathrm{d} \alpha}{(1-\mu) P}, \quad b_{L}(\mu P)=\frac{\int_{-P}^{\mu P} b_{L}(\beta) \mathrm{d} \beta}{(1+\mu) P}+\frac{\int_{\mu P}^{P} b_{R}(\beta) \mathrm{d} \beta}{(1-\mu) P} .
$$

The reason for choosing these particular conditions will become clear later, see (A5); for now notice only that for any $\phi$ eqs. (45b) define a unique $c$. The subset of $\mathcal{C}$ consisting of all functions $\phi$ for which $c=0$ is denoted $\stackrel{\circ}{\mathrm{C}}$.

Finally, the topology of our spacetime requires $\phi$ to have some periodicity properties. In order to satisfy them we take $a_{\curlywedge}$ and $b_{\curlywedge}$ to be periodic functions of $\alpha$ and $\beta$, respectively [13]. The period

$$
\text { of } a_{L} \text { and } b_{L} \text { is }(1+\mu) P, \quad \text { of } a_{R} \text { and } b_{R} \text { is }(1-\mu) P, \quad \text { of } a_{T} \text { and } b_{T} \text { is } 2 P \text {. }
$$

Now let us introduce the aforementioned functions of one variable. To this end denote by $\mathcal{F}$ the space of all continuous functions $A(x):[-P, P] \rightarrow \mathbb{C}$ (it is convenient to imagine $A$ as defined on the surface $t=0$; in doing so one, strictly speaking, must keep in mind that this surface lacks the points $x= \pm P, \mu P$, we shall omit this trivial reservation from now on) which

1. are smooth, except, perhaps, at the points $x=\mu P$ where the derivatives are allowed to have jump discontinuities;

2. satisfy the condition

$$
A(\mu P)=\frac{1}{(1+\mu) P} \int_{-P}^{\mu P} A(x) \mathrm{d} x+\frac{1}{(1-\mu) P} \int_{\mu P}^{P} A(x) \mathrm{d} x
$$

3. obey the "periodicity condition"

$$
A^{(n)}(-P)=A^{(n)}(\mu P-0), \quad A^{(n)}(\mu P+0)=A^{(n)}(P), \quad n=0,1 \ldots
$$


Each pair $A, B \in \mathcal{F}$ defines uniquely a function $\Psi(A, B) \in \stackrel{\complement}{ }$ in the following way: $a_{\curlywedge}$ are defined to be the extensions by periodicity, see (5c), of the functions, respectively,

$$
a_{T} \equiv A \quad \text { at } x \in(-P, P], \quad a_{L} \equiv A \quad \text { at } x \in(-P, \mu P], \quad a_{R} \equiv A \quad \text { at } x \in(\mu P, P] .
$$

The functions $b_{\curlywedge}$ are dealt with in exactly the same manner [the only difference is in the sign: $\left.\left.b_{\curlywedge}(\beta)\right|_{t=0}=B(-x)\right]$. Now $\left.\phi\right|_{\mathcal{M} \curlywedge}$ are built by (4) with $c=0$ and, finally, $\Psi(A, B)$ is defined to be the result of gluing together all three restrictions $\left.\phi\right|_{\mathcal{M}_{\curlywedge}}$.

Conversely, any $\phi \in \mathfrak{\complement}$ defines uniquely a pair $A, B \in \mathcal{F}$ such that $\Psi(A, B)=\phi$. This is done by decomposing $\phi$ into the right-moving and the left-moving parts $a(p)$ and $b(p)$, see (5a), and defining $A, B$ to be their restrictions

$$
\left.A(x(p)) \equiv a(p)\right|_{t=0},\left.\quad B(x(p)) \equiv b(p)\right|_{t=0} .
$$

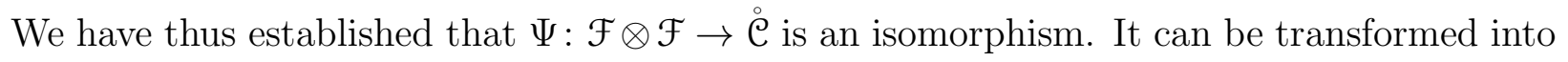
an isometry by an appropriate choice of the inner product in $\mathcal{F}$. Indeed, substituting the obvious expressions

$$
\left.\phi\right|_{t=0}(x)=A(x)+C(x),\left.\quad \dot{\phi}\right|_{t=0}(x)=A^{\prime}(x)-C^{\prime}(x), \quad \text { where } C(x) \equiv B(-x)
$$

into (2) one finds

$$
\begin{gathered}
\left(\phi_{1}, \phi_{2}\right)_{\mathrm{KG}}=<A_{1}, A_{2}>+<B_{1}, B_{2}>, \\
\text { where } \quad<F_{1}, F_{2}>\equiv i \int_{-P}^{P}\left[F_{2}^{*}(x) F_{1}^{\prime}(x)-F_{2}^{\prime *}(x) F_{1}(x)\right] \mathrm{d} x .
\end{gathered}
$$

2. Remark. The formulas (8) show that the singularity in $\mathcal{M}$ is harmless in the sense of [7]: there exists a unique solution to the wave equation for any Cauchy data fixed at a surface $t=t_{0} \leq 0$. It is, of course, this property that enables one to study QFT on $\mathcal{M}$ without adopting a number of $a d$ hoc assumptions.

\section{The choice of the Hilbert space and its basis}

Let $\left\{u_{L k}\right\}$ and $\left\{u_{R k}\right\}, k=1,2, \ldots$ be the sets of functions defined as follows:

$$
u_{L k}(x) \equiv\left\{\begin{array}{l}
(4 \pi k)^{-\frac{1}{2}} e^{-i k \xi} \text { at } x \in[-P, \mu P] \quad \text { i. e. } \quad \xi \in[-\pi, \pi] ; \\
(4 \pi k)^{-\frac{1}{2}} e^{-i k \pi}, \text { at } x \in[\mu P, P], \quad \text { where } \xi \equiv \frac{\pi}{1+\mu}\left(\frac{2}{P} x+1-\mu\right) .
\end{array}\right.
$$


and likewise

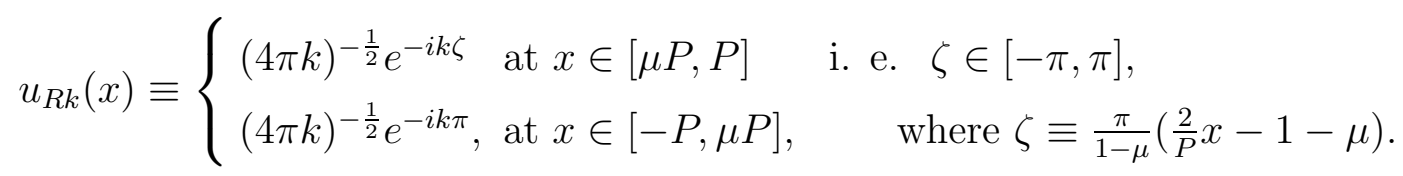

The set $\left\{u_{R k}, u_{L k}\right\}, k=1,2, \ldots$ is orthonormal w.r.t. the scalar product $<,>$ defined in (10) and we denote by $\mathcal{H}_{F}$ the (auxiliary) Hilbert space obtained by declaring that set to be a basis.

The modes $\phi_{k}$ we are after are now defined as

$$
\phi_{Q k} \equiv\left\{\begin{array}{ll}
\Psi\left(u_{Q k}, 0\right), & k>0 \\
\Psi\left(0, u_{Q|k|}^{*}\right), & k<0
\end{array}, \quad \text { where } Q \equiv R, L\right.
$$

(i. e. $\phi_{Q k}$ are obtained from $u_{Q k}$ by replacing $x \rightarrow-\beta=x-t$ for $k<0$ and $x \rightarrow-\rightarrow=x+t$ for $k>0$ and extending the resulting functions by periodicity). So, every mode is at first a harmonic wave that moves in the corresponding leg (left or right depending on whether the subscript is $L$ or $R$ ) to the left or to the right depending on the sign of $k$. In the trunk, however, the behavior of the mode becomes more exotic. It is just a constant here except in a spiral strip bounded by two null geodesics emanating from the crotch. Within that strip the mode is still a piece of a harmonic wave whose fronts are just those geodesics. The mode, though continuous - which enables the SET to remain bounded, contrary to the AD conjecture - is not smooth. So, one does not expect the energy to be conserved, but this is natural for a non-static spacetime.

3. Example. Assume $Q=L, k=-3$. Then we, first, find that $u_{Q|k|}^{*}$ is the function (we again perceive its domain as the segment $t=0$ with the values at the missing points $x= \pm P, \mu P$ being defined by continuity) equal to $\sqrt{12 \pi} e^{3 i \xi(x)}$ at $x \in[-P, \mu P]$ and $-\sqrt{12 \pi}$ otherwise. Correspondingly, for every $p \in \mathcal{M}$ we define $\phi_{L,-3}(p)$ to be equal to

$$
\Psi\left(0, u_{L 3}^{*}\right)= \begin{cases}\sqrt{12 \pi} e^{3 i \xi\left(x_{p}\right)}, & \text { at } x_{p} \in[-P, \mu P] \\ -\sqrt{12 \pi}, & \text { otherwise. }\end{cases}
$$

where $x_{p}$ is the $x$-coordinate of the point at which the null $\alpha$-directed geodesic through $p$ meets the segment $t=0$. Whence, in particular, $\phi_{L,-3}=-\sqrt{12 \pi}$ in the entire non-hatcfed region in figure 2. To write down the explicit expression for $\phi_{L,-3}$ in the remaining part of $\mathcal{M}$ one replaces $x \rightarrow-\beta=x-t$ in the relevant $u$ and specifies the periodicity condition

$$
\phi_{L,-3}=\sqrt{12 \pi} e^{\frac{3 i \pi(\mu-1-2 \beta / P)}{1+\mu}}, \quad \beta \in \begin{cases}{[-\mu P+P(1+\mu) n, P+P(1+\mu) n],} & \text { in } \mathcal{M}_{L} \\ {[-P+P n, P+P n],} & \text { in } \mathcal{M}_{T}\end{cases}
$$




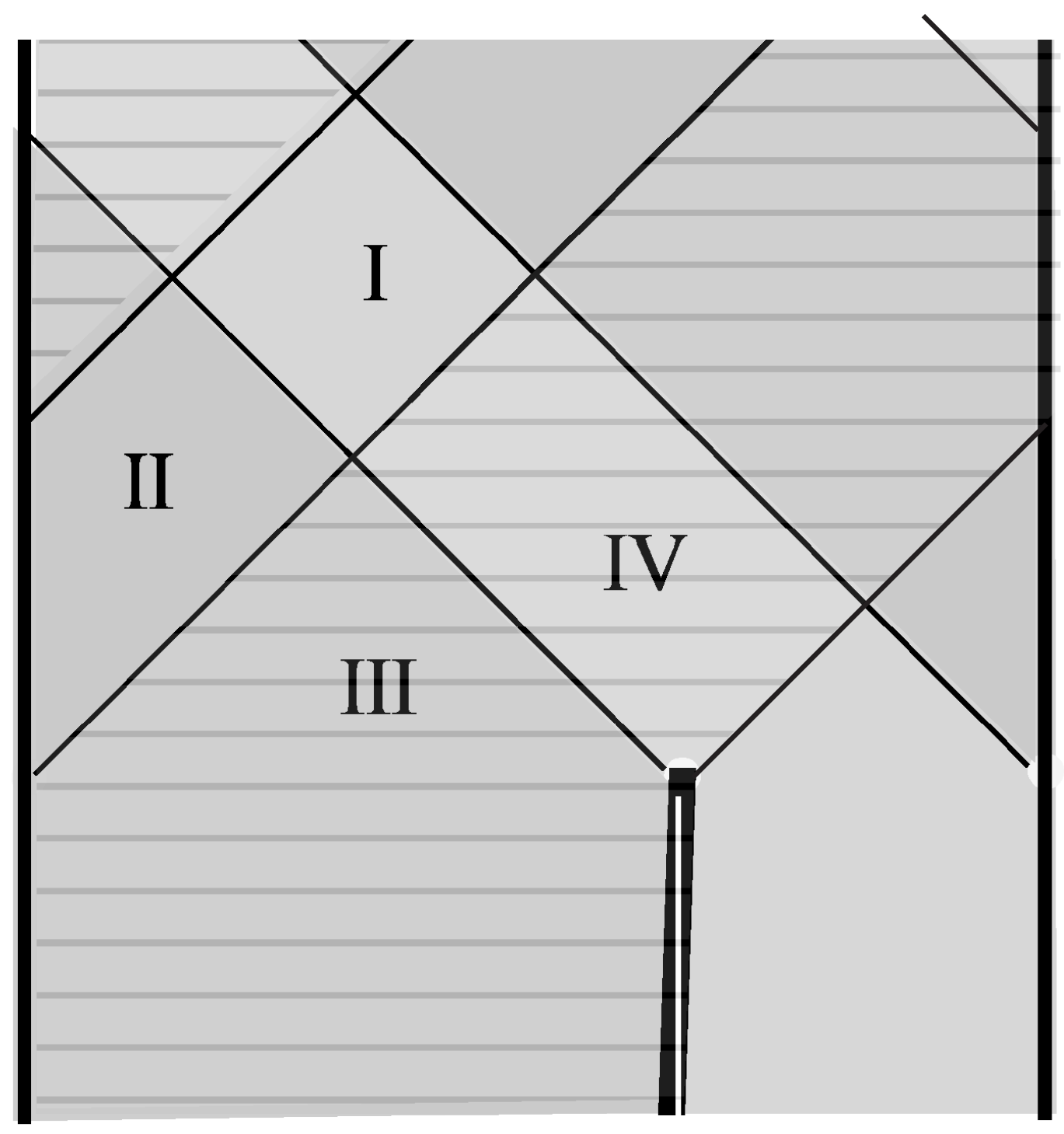

FIG. 2: The slanted rays are the null geodesics at which the derivatives of functions of $\stackrel{\circ}{\complement}$ are allowed to have jumps. In the dark and in the hatched regions $\phi_{R k}$ with, respectively, positive and negative $k$ are constant. In the complements to those regions $\phi_{L k}$ with $k$ of the same sign are constant.

4. Remark. The problem obtained by restricting the consideration to the right (for definiteness) leg and choosing the vacuum to be that defined by the set of modes $\left\{\phi_{R k}\right\}$ is well studied, see [10]. The index $Q$ absent in that case may seem to double "the number" of modes (for $\mu=0$, say). Note, however, that the separation between the frequencies of the modes in the trousers is twice that between the ones in the cylinder. 
Now let the sought-for Hilbert space $\mathcal{H}$ be defined as the completion of the linear span of the set

$$
\left\{\phi_{R k}\right\} \cup\left\{\phi_{L k}\right\}, \quad k=1,2 \ldots
$$

by the norm generated by the scalar product (2). That it is indeed a scalar product (which requires positive definiteness) follows from the easily verified equality

$$
\left(\phi_{Q k}, \phi_{\check{Q} n}\right)_{\mathrm{KG}}=\delta_{k n} \delta_{\check{Q} Q}, \quad \text { for all } \check{Q}, Q \quad k, n=1,2 \ldots
$$

According to the plan outlined in the end of section $\llbracket \mathrm{A}$ it only remains to prove (3), which is done in the Appendix.

\section{The vacuum SET}

The (non-renormalized) vacuum expectation value of the SET is $<T_{\mu \nu}>=\sum_{n} \hat{T}_{\mu \nu}\left[\phi_{n}\right]$, where

$$
\hat{T}_{\mu \nu}[f] \equiv f,_{\mu} f^{*}{ }_{\nu}-\frac{1}{2} \eta_{\mu \nu} \eta^{\kappa \lambda} f_{, \kappa} f^{*}{ }_{\lambda}
$$

and the summation is over all modes [10]. The series diverges of course and to renormalize the result one introduces a cut-off factor into the divergent sum by replacing $t--t-i \delta$ and lets $\delta \rightarrow 0$ at the end of the calculation, that is, after subtracting the limit at $P \rightarrow \infty$. Thus,

$$
<\mathbf{T}_{\mu \nu}^{\mathrm{ren}}>=T_{>\mu \nu}^{L}+T_{<\mu \nu}^{L}+T_{>\mu \nu}^{R}+T_{<\mu \nu}^{R}
$$

where

$$
T_{\lessgtr \mu \nu}^{Q} \equiv \lim _{\delta \rightarrow 0}\left[\left(1-\lim _{P \rightarrow \infty}\right) \sum_{n \lessgtr 0} \hat{T}_{\mu \nu}\left[\phi_{Q n}, \delta\right]\right],
$$

where $\hat{T}_{\mu \nu}\left[\phi_{Q n}, \delta\right]$ is the result of the substitution $t \rightarrow-\rightarrow-i \delta$ into $\hat{T}_{\mu \nu}\left[\phi_{Q n}\right]$. Let us find the four terms in turn. In the coordinate basis

$$
\hat{T}_{\mu \nu}\left[e^{-i C(t-x)}\right]=C^{2}\left(\begin{array}{cc}
1 & -1 \\
-1 & 1
\end{array}\right)
$$


where $C$ is a real constant [note that the second term of (13) vanishes]. Correspondingly, the term $T_{<\mu \nu}^{L}$, when non-zero (i. e. in the hatched region in figure 1) is

$$
\begin{aligned}
\lim _{\delta \rightarrow 0}\left\{\left(1-\lim _{P \rightarrow \infty}\right) \sum_{n<0} \hat{T}_{\mu \nu}\left[\frac{\exp \left\{i|n| \frac{\pi}{1+\mu}\left(\mu-1-\frac{2}{P}(\beta-i \delta)\right)\right\}}{\sqrt{4 \pi|n|}}\right]\right\} & =-\frac{\pi}{12 P^{2}(1+\mu)^{2}}\left(\begin{array}{cc}
1 & -1 \\
-1 & 1
\end{array}\right) .
\end{aligned}
$$

The term $T_{>\mu \nu}^{L}$ differs from $T_{<\mu \nu}^{L}$ in two respects: 1) it vanishes in the light gray region and 2) the exponents in the expression for modes depend now on $t+x$ instead of $t-x$, which changes the sign of the $t x$ components. Correspondingly,

$$
T_{>\mu \nu}^{L}=-\frac{\pi}{12 P^{2}(1+\mu)^{2}}\left(\begin{array}{ll}
1 & 1 \\
1 & 1
\end{array}\right) \quad \text { in the dark region and }=0 \text { otherwise. }
$$

Changing the sign of $\mu$ we find

$$
\begin{gathered}
T_{<\mu \nu}^{R}=-\frac{\pi}{12 P^{2}(1-\mu)^{2}}\left(\begin{array}{cc}
1 & -1 \\
-1 & 1
\end{array}\right) \quad \text { in the non-hatched region and }=0 \text { otherwise. } \\
T_{>\mu \nu}^{R}=-\frac{\pi}{12 P^{2}(1-\mu)^{2}}\left(\begin{array}{ll}
1 & 1 \\
1 & 1
\end{array}\right) \quad \text { in the light region and }=0 \text { otherwise. }
\end{gathered}
$$

Summing these terms up one finally gets (the description and numbering refer to figure 2):

light non-hatched regions I $\quad<\mathbf{T}_{t t}^{\mathrm{ren}}>=<\mathbf{T}_{x x}^{\mathrm{ren}}>=-\frac{\pi}{6 P^{2}(1-\mu)^{2}}$,

$$
<\mathbf{T}_{t x}^{\mathrm{ren}}>=<\mathbf{T}_{x t}^{\mathrm{ren}}>=0
$$

dark hatched regions III $\quad<\mathbf{T}_{t t}^{\mathrm{ren}}>=<\mathbf{T}_{x x}^{\mathrm{ren}}>=-\frac{\pi}{6 P^{2}(1+\mu)^{2}}$,

$$
<\mathbf{T}_{t x}^{\mathrm{ren}}>=<\mathbf{T}_{x t}^{\mathrm{ren}}>=0
$$

light hatched regions IV $\quad<\mathbf{T}_{t t}^{\mathrm{ren}}>=<\mathbf{T}_{x x}^{\mathrm{ren}}>=-\frac{\pi}{6 P^{2}} \frac{1+\mu^{2}}{\left(1-\mu^{2}\right)^{2}}$,

$$
<\mathbf{T}_{t x}^{\mathrm{ren}}>=<\mathbf{T}_{x t}^{\mathrm{ren}}>=-\frac{\pi}{3 P^{2}} \frac{\mu}{\left(1-\mu^{2}\right)^{2}} ;
$$




$$
\begin{aligned}
\text { and dark non-hatched regions II } \quad<\mathbf{T}_{t t}^{\mathrm{ren}}>=<\mathbf{T}_{x x}^{\mathrm{ren}}>=-\frac{\pi}{6 P^{2}} \frac{1+\mu^{2}}{\left(1-\mu^{2}\right)^{2}}, \\
<\mathbf{T}_{t x}^{\mathrm{ren}}>=<\mathbf{T}_{x t}^{\mathrm{ren}}>=\frac{\pi}{3 P^{2}} \frac{\mu}{\left(1-\mu^{2}\right)^{2}} .
\end{aligned}
$$

Thus, in the reference frame of a free falling observer the stress-energy tensor is componentwise bounded even though in the general case it suffers discontinuities of the first kind. If the legs are equal $(\mu=0)$ there is no "thunderbolt" - the SET is perfectly regular.

\section{Acknowledgements}

This work was supported by RNP Grant No. 15-02-06-818.

\section{Appendix A: The relation between the classical and the quantum spaces}

By construction $\stackrel{\circ}{\mathcal{C}}$ is dense in $\mathcal{H}\left(\right.$ since all $\left.\phi_{Q k} \in \stackrel{\complement}{\mathrm{C}}\right)$. In this appendix a converse, in a sense, property (3) is established.

For an arbitrary function $A \in \mathcal{F}$ let us prove that

$$
A=A^{+}+A^{-}, \quad \text { where } A^{+},\left(A^{-}\right)^{*} \in \mathcal{H}_{F} .
$$

Obviously, this implies (3) for all $f$ of the type $f=\Psi(A, 0)$ [it suffices to set $f^{ \pm}=\tilde{\Psi}\left(A^{ \pm}, 0\right)$ where $\tilde{\Psi}$ is the extension by continuity of $\Psi$ to the entire $\left.\mathcal{H}_{F} \otimes \mathcal{H}_{F}\right]$. The case of $f=\Psi(0, B)$ is perfectly analogous and $f$ of the general type is just the sum of those two plus a constant. So, the validity of (A1) will prove (3).

Proof of (A1). The mode $u_{1}$ and the function $A$ are smooth except at $x=\mu P$ where either of them has a-non-zero in the case of $u_{1}$ - jump in its first derivative see [(17)],

$$
w^{\prime}(\mu P+0)-w^{\prime}(\mu P-0)=w^{\prime}(P)-w^{\prime}(-P), \quad \text { where } w \equiv A, u_{1} .
$$

So, we can find a continuously differentiable linear combination

$$
A_{1} \in C^{1}, \quad A_{1}(P)=A_{1}(-P), \quad A_{1}^{\prime}(P)=A_{1}^{\prime}(-P), \quad \text { where } A_{1} \equiv A-C_{u} u_{1}, C_{u}=\text { const. }
$$

Evidently, (A1) is true iff it is true with $A$ replaced by $A_{1}$. Thus it involves no loss of generality to assume that $A \in C^{1}$. 
Now, consider the Fourier coefficients

$$
\left.F_{L, k} \equiv \frac{1}{2 \pi} \int_{-\pi}^{\pi} A\right|_{x \in[-P, \mu P]}(x(\xi)) e^{i k \xi} \mathrm{d} \xi,\left.\quad F_{R, k} \equiv \frac{1}{2 \pi} \int_{-\pi}^{\pi} A\right|_{x \in[\mu P, P]}(x(\zeta)) e^{i k \zeta} \mathrm{d} \zeta .
$$

of (the restrictions of) $A$. By [11, $\left.\mathrm{n}^{\circ} 708\right]$ it follows from the continuous differentiability [14] of $A$ that

$$
F_{Q, k}=O\left(k^{-3}\right)
$$

This rate of convergence implies that for some functions $X_{Q}^{ \pm}$

$$
\begin{array}{r}
S_{Q}^{+}\left(k_{0}\right) \equiv \sum_{k=1}^{k_{0}} F_{Q, k} u_{Q k} \sqrt{4 \pi|k|} \quad \text { and } S_{Q}^{-}\left(k_{0}\right) \equiv \sum_{k=-k_{0}}^{1} F_{Q, k}^{*} u_{Q,-k} \sqrt{4 \pi|k|} \text { converge } \\
\text { uniformly to, respectively, } X_{Q}^{+} \text {and } X_{Q}^{-}
\end{array}
$$

and

$$
\left[S_{Q}^{ \pm}\left(k_{0}\right)\right]^{\prime} \text { converges uniformly to } Y_{Q}^{ \pm} \equiv\left[X^{ \pm}{ }_{Q}\right]^{\prime}
$$

The expression $S_{L}^{+}+S_{L}^{-*}+\frac{1}{(1+\mu) P} \int_{-P}^{\mu P} A(x) \mathrm{d} x$ on the interval $x \in[-P, \mu P]$ is a partial sum of the Fourier series of $\left.A\right|_{x \in[-P, \mu P]}$. So, it converges to the said function there while the first two terms tend, respectively, to $X_{L}^{+}$and $X_{L}^{-*}$ ). On the other interval (i. e. at $x \in[\mu P, P])$ it converges to $A(\mu P)$. Similar considerations apply to $S_{R}^{+}+S_{R}^{-*}$ and hence

$$
\begin{array}{r}
\left.A\right|_{x \in[-P, P]}=X_{L}^{+}+X_{L}^{-*}+\frac{1}{(1+\mu) P} \int_{-P}^{\mu P} A(x) \mathrm{d} x+X_{R}^{+}+X_{R}^{-*}+\frac{1}{(1-\mu) P} \int_{\mu P}^{P} A(x) \mathrm{d} x-A(\mu P) \\
=\left(X_{L}^{+}+X_{R}^{+}\right)+\left(X_{L}^{-}+X_{R}^{-}\right)^{*}
\end{array}
$$

[the last equality follows from ([6)]. Comparing this with (A1) we see that the latter is proven once we show that

$$
X_{Q}^{+}, X_{Q}^{-*} \in \mathcal{H}_{F}
$$

So, recall that by construction $S_{Q}^{+}, S_{Q}^{-*} \in \mathcal{H}_{F}$, see (A3). At the same time by (A33) and (A4)

$$
\max \left|X_{Q}^{ \pm}-S_{Q}^{ \pm}\left(k_{0}\right)\right|, \max \left|X_{Q}^{\prime \pm}-S_{Q}^{\prime \pm}\left(k_{0}\right)\right| \underset{k_{0} \rightarrow \infty}{\longrightarrow} 0
$$

and hence $S_{Q}^{+}$and $S_{Q}^{-*}$ converge to, respectively, $X_{Q}^{+}$and $X_{Q}^{-*}$ in the metric of $\mathcal{H}_{F}$, see (10). Thus, the containment (A6) follows from the completeness of the Hilbert space $\mathcal{H}_{F}$.

[1] A. Anderson and B. DeWitt, Foundations of Physics 16, 91 (1986). 
[2] A. Strominger in Les Houches Summer School, Les Houches, 1994.

[3] F. Dowker and S. Surya, Phys.Rev. D 58, 124019 (1998).

[4] R. D. Sorkin Consequences of spacetime topology, in A. Coley, F. Cooperstock, B. Tupper (eds.), Proceedings of the Third Canadian Conference on General Relativity and Relativistic Astrophysics held May, 1989, Victoria, Canada (World Scientific, 1990).

[5] A. O. Barvinsky, JCAP 9, 033 (2012).

[6] C. A. Manogue, E. Copeland and T. Dray, Pramana J. Phys. 30, 279 (1988).

[7] A. Ishibashi and A. Hosoya, Phys. Rev. D66, 104016 (2002).

[8] J. L. Friedman and M. S. Morris, Commun. Math. Phys. 186, 495 (1997).

[9] C. L. Duston, Class. Quant. Grav. 29, 205015 (2012).

[10] N. D. Birrel and P. C. V. Davies Quantum fields in curved space (Cambridge, Cambridge University Press, 1982)

[11] G. M. Fichtenholz Course in differential and integral calculus. (In Russian) v. III (Moscow, 1947).

[12] With our choice of the future direction the mode's labels 'in' and 'out' must be interchanged.

[13] The term proportional to $\alpha+\beta$ is duly smooth on $\mathcal{M}$ without being a sum of such periodic functions; it is excluded, however, by the boundedness of $\phi$.

[14] And equality $A_{1}^{\prime}(P)=A_{1}^{\prime}(-P)$. Actually, there are a few more requirements, but they are automatically satisfied by functions of $\mathcal{C}$. 